Revista Científica de FAREM-Estelí

Medio ambiente, tecnologia y desarrollo humano

Año 10 | Núm. 39| Julio-septiembre, 2021

ISSN: 2305-5790

https://rcientificaesteli.unan.edu.ni

DOI: https://doi.org/10.5377/farem.v10i39.12614

\section{Vivencia familiar de los hermanos de niños diagnosticados con Trastornos del Espectro Autista. Estelí, Nicaragua}

\section{Family experience of siblings of children diagnosed with Autism Spectrum Disorders. Estelí, Nicaragua}

\section{Juana Sarahi Reyes Castillo}

Egresada de la carrera de Psicología. Universidad Nacional Autónoma de

Nicaragua UNAN Managua. Facultad Regional Multidisciplinaria de Estelí. FAREM Estelí.

sarahireyescastillo07@gmail.com

\section{Dayana Massiel Noguera Valle}

Egresada de la carrera de Psicología. Universidad Nacional Autónoma de

Nicaragua UNAN Managua. Facultad Regional Multidisciplinaria de Estelí. FAREM

Estelí.

dayananoguera04@gmail.com

\section{Jeyson Ramón Torres Escoto}

Egresado de la carrera de Psicología. Universidad Nacional Autónoma de

Nicaragua UNAN Managua. Facultad Regional Multidisciplinaria de Estelí. FAREM

Estelí.

jeytorres2017@gmail.com

\section{Franklin Jesús Solís Zúniga}

Asesor y docente de la carrera de Psicología. Universidad Nacional Autónoma de Nicaragua UNAN Managua. Facultad Regional Multidisciplinaria de Estelí. FAREM Estelí.

frankzuni@gmail.com

\section{RESUMEN}

El Trastorno del Espectro Autista (TEA) se encuentra dentro de los trastornos del neurodesarrollo y se caracteriza por las alteraciones relacionadas con la comunicación y la interacción social, así como por presentar intereses fijos y conductas repetitivas (Kids Health, 2019). El objetivo de este estudio ha sido analizar la vivencia familiar de los hermanos de niños diagnosticados con Trastornos del Espectro Autista, en la ciudad de Estelí. La metodología que se planteó fue en base a un estudio cualitativo de tipo exploratorio y descriptivo con un enfoque fenomenológico. El tipo de muestreo es no probabilístico intencional, se seleccionaron cinco familias que cumplieran con el criterio de muestreo. Las técnicas utilizadas fueron entrevista en profundidad y test de la familia. Los resultados demuestran que la vivencia familiar de los hermanos de niños diagnosticados con TEA, realizan el cumplimiento de deberes en la vida de su hermano tal como: cuidador, protector y facilitador de las
RECIBIDO

$14 / 05 / 2021$

ACEPTADO

$04 / 10 / 2021$

\section{PALABRAS CLAVE}

Vivencia familiar; Trastorno del Espectro Autista; rol de los hermanos; repercusiones psicosociales; estrategias de intervención. 
relaciones sociales. Para los hermanos de niños autistas no todo ha sido negativo, a pesar del cambio que ha significado el nuevo diagnóstico en la familia, muestran una mayor empatía, tolerancia al estrés, capacidad para adaptarse y mayor sentido de responsabilidad de cuidado de la familia. Se demuestra que los padres y madres de familia han dado mucha responsabilidad a sus hijos sobre el cuido a su hermano con autismo. La mayoría de los hermanos que ejercen el rol de mamá o papá en el niño autista, se debe a la ausencia de la figura paterna o materna, este dato es uno de los factores que más inciden en el apego y sobreprotección hacia el hermano autista. Finalmente, se propone una estrategia de intervención psicológica aplicada a los hermanos de niños autistas, con el fin de mejorar la adaptación y disminuir su estrés.

\section{ABSTRACT}

Autism Spectrum Disorder (ASD) is within the neurodevelopmental disorders and is characterized by alterations related with the communication and social interaction, as well as by presenting fixed interests and repetitive behaviors (Kids Health, 2019). The objective of this study was to analyze the family experience of the siblings of children diagnosed with Autism Spectrum Disorder, in the city of Estelí. The methodology used was based on an exploratory and descriptive qualitative study with a phenomenological approach. The type of sampling was non-probabilistic and intentional, five families were selected that met the sampling criteria. The techniques used were In-depth interview and family test. The results show that the family experience of the siblings of children diagnosed with ASD, perform the fulfillment of duties in the life of their siblings such as: caregivers, protector and facilitator of the social relationship. For the siblings of autistic children not everything has been negative, in spite of the change that the new diagnosis has meant for the family, they show for the family greater empathy, tolerance to stress and capacity to adapt and a greater sense of responsibility for the care of the family. It is demonstrated that the parents have given their children a great deal of responsibility for the care of their siblings with autism. Most of the siblings who play the role of mother and father in the autistic child, is due to the absence of a father or mother figure, this fact in one of the factors that most affect attachment and overprotection toward the autistic siblings. Finally, a psychological intervention strategy applied to the siblings' autistic children is proposed in order to improve their adaptation and reduce their stress.

\section{KEYWORDS}

Family experience; Spectrum Autism Disorder; sibling role; psychosocial repercussions; intervention strategies. 


\section{INTRODUCCIÓN}

La presente investigación cabe dentro de la línea número uno Salud Pública, tema: Salud Mental, abordando el tema Vivencia familiar de los hermanos de niños diagnosticados con Trastornos del Espectro Autista, en la ciudad de Estelí, el cual se conoce como una afección neurológica y de desarrollo que comienza en la niñez y dura toda la vida. Afecta cómo una persona se comporta, interactúa con otros, se comunica y aprende. Se le llama trastorno de espectro porque diferentes personas con TEA pueden tener una gran variedad de síntomas distintos. Estas pueden tener problemas para hablar con las demás personas y es posible que no las miren a los ojos cuando les hablan. Además, pueden tener intereses limitados y comportamientos repetitivos. (Peral Feliz, 2013)

La familia es considerada como la unidad de apoyo social compuesta por diferentes miembros, donde cada uno de ellos aporta en la formación de los más pequeños. La noticia sobre un diagnóstico de este tipo puede generar un impacto emocional muy fuerte ante la aceptación de la discapacidad entre ellos pueden experimentar estadios de shock, negación, tristeza, ira, ansiedad, adaptación y reorganización, así mismo cada familia vivirá este cambio de manera diferente por sus propias características como: la formación académica, los estados de salud física y mental, los valores, la cultura y la resiliencia familiar. (Perez Castañeda \& Verdugo Alonso, 2006)

De aquí parte la importancia de indagar sobre la vivencia familiar de los hermanos con niños/as diagnosticados con Trastornos del Espectro Autista, tomando en cuenta que la relación entre los hermanos es una base fundamental para el desarrollo social del niño, así mismo representa la complicidad, el compañerismo, la formación de la empatía y colaboración entre sus pares.

Se retoman teorías para explicar de manera científica la dinámica familiar de los niños diagnosticados con TEA tal como es la teoría sistémica, un modelo propuesto por Freixa (1993) basado en la teoría sistémica para comprender el funcionamiento de la familia ante la discapacidad. Este modelo permite explicar algo que debemos grabarnos a fuego en nuestro pensamiento a la hora de valorar el impacto que genera el autismo de uno de los hijos en la familia: no todas responden igual. Freixa (1993) plantea que los efectos de la discapacidad van a depender de las propiedades del sistema tales como las características de cada uno de los miembros, la naturaleza de sus interacciones, el nivel de cohesión, sus roles dentro de la familia y la etapa del ciclo vital en la que se encuentre cada uno de ellos.

La realidad familiar es diferente antes incluso del diagnóstico. Algunas de las diferencias tienen que ver con la incertidumbre que plantea el desarrollo 
disarmónico del niño con TEA, y la ausencia o retraso de un diagnóstico y pronóstico claros (Martínez Martín \& Bilbao León, 2008). A esto se le sumarían los efectos de las manifestaciones del TEA sobre la convivencia diaria de la familia (las dificultades para comunicarse, los problemas de conducta o la dependencia que presenta el niño).

Según Meynckens-Fourez (1999) citado por Peral Feliz (2013), la relación fraterna ejerce, al menos, tres funciones: apego, suplencia parental y aprendizaje de los papeles sociales y cognitivos. López (1984) señala que el apego persiste a lo largo de la vida y que en la edad adulta se manifiesta por medio del mantenimiento de una relación periódica con los hermanos. Para Milevsky (2005) el apoyo fraterno es compensatorio, sobre todo ante la falta de los padres y amigos.

Moguillansky y Vorchheimer (2001) citado por Peral Feliz (2013), otorgan, al vínculo fraterno, características de modelo del sentimiento de pertenencia, lo que va a repercutir en las futuras relaciones sociales. La importancia del hermano en el desarrollo emocional resalta el papel y la función del vínculo fraterno en la relación familiar Vivona (2007). La función fraterna enfatiza la necesidad de la participación del semejante en la formación de la personalidad.

Más allá del vínculo y de la función, Kancyper (2004), citado por Peral Feliz (2013), defiende la existencia del llamado complejo fraterno y lo define como un conjunto organizado de deseos hostiles y amorosos que el niño experimenta respecto de sus hermanos. El niño cela intensamente a sus hermanos y hermanas porque son sus rivales en el amor de los padres. Sin embargo, como también los ama, surgen fuertes conflictos entre los impulsos agresivos y los sentimientos de amor. Esto provoca culpa y origina nuevos deseos de hacer reparaciones, mezcla los sentimientos que tienen gran influencia no solo en la relación entre hermanos, sino también, ya que las relaciones humanas obedecen el mismo patrón, en la actitud social, el amor, la culpa y los futuros deseos de reparar.

Cada hermano, desde su propia situación, incorpora, además, diversas protestas fraternas. Landolfi (2012) citado por Peral Feliz (2013) describe la culpa fraterna, especialmente aquella que se experimenta hacia el hermano cuyo destino ha sido aciago. En el caso de las patologías graves como el autismo, además de con la culpa, el hermano puede responder con negación, sumisión masoquista $u$ otras reacciones defensivas.

Como antecedentes relevantes de esta investigación se encontró un estudio de Peral Feliz (2013), titulado: Hermanos de personas con el trastorno del espectro autista. Este estudio se realizó con el principal objetivo de profundizar en el punto de vista de los hermanos y obtener información sobre sus percepciones, sentimientos y pensamientos subjetivos acerca de tener un hermano con autismo. Se llegó a la conclusión tentativa de que 
la experiencia de tener un hermano con autismo difiere en ciertos aspectos de la experiencia de crecer con un hermano con desarrollo típico o con otro tipo de discapacidad. Esta conclusión está basada en el hecho de que los hermanos suelen expresar que lo más difícil para ellos son sobre todo los problemas de comportamiento y las dificultades en la comunicación e interacción social del hermano con autismo.

La Universitat Jaume I Valencia España realizó un estudio por González (2018) con el tema Proyecto de apoyo a hermanos de niños diagnosticados con Trastorno de espectro autista. Este proyecto se realizó con el objetivo principal de plantear un programa de intervención que mejore la vida de los hermanos de TEA y que esto repercuta en la mejor calidad de vida de todos los miembros de la familia de entender qué es el autismo y cuáles son sus características. Para crear un espacio de confianza entre personas que comparten la circunstancia de tener un hermano con TEA, donde expresar sentimientos que produce esta situación pudiendo crear una red social de apoyo.

Está claro una vez hecho todo este recorrido, que no se puede afirmar que el tener un hermano con autismo conlleve un desajuste en el desarrollo psicológico de la persona, pero lo que sí es cierto es que esto trae consigo una serie de sentimientos tanto positivos como negativos que no podemos ignorar, como pueden ser: la culpa, la responsabilidad, la resiliencia, los celos y la angustia en relación a su hermano.

Es sabido que toda enfermedad o trastornos graves y crónicos, provocan un impacto importante en las personas más cercanas al sujeto afectado. Por este motivo, las familias de personas que presentan un trastorno del espectro autista (trastorno del neurodesarrollo crónico y con frecuencia severo) suelen sufrir consecuencias derivadas de la convivencia. El impacto en las familias se caracteriza, sobre todo, por la presencia de estrés permanente, que, en el caso del TEA, suele ser superior al de las familias con niños/as que presentan una discapacidad intelectual sin autismo. (Peral Feliz, 2013).

\section{MATERIALES Y MÉTODOS}

Esta investigación pertenece al paradigma interpretativo de tipo cualitativo, se basa en métodos de recolección de datos no estandarizados. No se efectúa una medición numérica, por lo cual el análisis no es estadístico. Es una investigación con diseño fenomenológico, ya que su propósito principal es explorar, describir y comprender las experiencias de las personas con respecto a un fenómeno y descubrir los elementos en común de tales vivencias (Hernandez, 2014). Según su alcance temporal es de corte transversal, porque 
se llevó a cabo entre el período de agosto- diciembre 2020, específicamente en la ciudad de Estelí.

En este estudio se busca describir la vivencia familiar de los hermanos de niños diagnosticados con Trastornos del Espectro Autista, en la ciudad de Estelí y cabe en la línea de investigación Número uno Salud Mental tema: Comportamiento Psicosocial.

\section{Población objeto de estudio}

Este trabajo investigativo se realizó en la ciudad de Estelí, cuenta con 226,604 habitantes y está dividida en tres distritos. En la ciudad de Estelí existen diferentes centros en donde se atienden niños con TEA y otros diagnósticos entre ellos están: Fundación Pedagógica Cristal, Centro de Intervención EduTerapéutico y Escuela de Educación Especial Héroes y Mártires de Ayapal Estelí.

Esta investigación se llevó a cabo con cinco familias de niños/as diagnosticados con Trastorno del Espectro Autista del municipio de Estelí. Los sujetos participantes de esta investigación fueron los hermanos de niños con Trastorno del Espectro Autista de la ciudad de Estelí. Totalizando a cinco familias diferentes.

Esta investigación se llevó a cabo con cinco hermanos de niños diagnosticados con Trastorno de Espectro Autista que se han atendidos en diferentes centros tales como la Fundación Cristal, el Centro de Intervención Edu- Terapéutico $\mathrm{CIE}$, y la Escuela Educación Especial Mártires de Ayapal, la muestra la representarán los hermanos de estos niños entre las edades de 10-13 años. El tipo de muestreo es no probabilístico intencional, la elección de los elementos no depende de la probabilidad, sino de causas relacionadas con las características de la investigación o de quien hace la muestra. (Hernandez Sampieri, Fernandez Collado, \& Baptista Lucio, 2007)

Para seleccionar la muestra se definieron los siguientes criterios:

- Hermanos biológicos de niños con Trastorno del Espectro Autista.

- Hermanos que convivan con los niños con Trastorno del Espectro Autista.

- Hermanos menores de 13 años.

- Padres y madres que convivan con sus hijos.

- Participación voluntaria en el estudio.

- Consentimiento informado de los padres de familia.

\section{Técnicas e instrumentos de recolección de datos:}

La recolección de los datos consiste en obtener las perspectivas y puntos de vistas de los participantes (sus emociones, experiencias, significados y otros aspectos subjetivos tal es; el caso de la vivencia familiar de los hermanos de niños con TEA) (Hernandez Sampieri, Fernandez Collado, \& Baptista Lucio, 
2007). Para el cumplimiento de los objetivos planteados en esta investigación, se utilizaron diferentes técnicas tales como: entrevista en profundidad y el test del dibujo de la familia.

La entrevista a profundidad es un conjunto de preguntas previamente elaboradas que determinen la estructura y contenido de un tema. El orden de sucesión de las preguntas, así como su formulación no están establecidos de manera rígida. (Piura Lopez, 2000). Se abordaron aspectos relacionados a la vivencia de los hermanos de niños autistas, se analizó la percepción acerca de tener un hermano autista, el rol que ejercen y se identificaron las repercusiones psicosociales que estos experimentan.

El test de la familia es una prueba proyectiva de personalidad, que se administra a partir de cinco años y hasta los dieciséis. En ella se analiza la percepción que tiene el niño de su familia y del lugar que ocupa en ella. El test de la familia fue creado por Porot (1952) y basado en el dibujo libre que tanto gusta a los niños. (Meneu, 2019)

\section{Procesamiento y análisis de datos}

Para el procesamiento del análisis de los resultados en los instrumentos que fueron aplicados a hermanos y padres de niños autistas que cumplen con los criterios del estudio, se analizaron y procesaron a través de un análisis cualitativo. Los datos obtenidos de las distintas técnicas aplicadas para la obtención de datos fueron procesados, analizados e interpretados en función de los objetivos formulados y haciendo uso del programa de Word. El análisis de datos cualitativos de la entrevista en profundidad, se realizó a través de la transcripción fiel la cual se organizó en matrices.

Para el análisis del dibujo de la familia se hizo a través de la interpretación de Lovis Corman procesando la información en una matriz, por medio del programa de Word respetando las normas para su análisis finalizando con una conclusión general sobre los aspectos más relevantes en la prueba.

Para la triangulación de instrumentos se procesó la información a través de una matriz por medio del programa de Word en donde por categorías se seleccionó las respuestas que más coincidían, finalizando con una conclusión sobre las repercusiones psicosociales.

Para llevar a cabo esta investigación fue necesario el enlace con los diferentes Centros en donde son atendidos los niños con TEA, quienes colaboraron en la localización de las diferentes familias que cumplieran con los criterios de muestra establecidos, y el consentimiento de padres, madres o tutores de niños/as (hermanos) que colaboraron en el estudio. También se sustituyeron los nombres verdaderos de cada uno de los participantes por una letra y se les nomino $\mathrm{H}$ a hermano uno, $\mathrm{M}$ a mamá y $\mathrm{P}$ papá poniendo en práctica la ética profesional como investigador y cuidando de la identidad de los miembros. 


\section{RESULTADOS Y DISCUSIÓN}

\section{Caracterización de la muestra}

Los hermanos sujetos de muestra en el estudio en su mayoría pertenecen al sexo femenino y dos al sexo masculino, en cuanto a edad se encuentran dos hermanos de 13 años, un hermano de 11 años y dos hermanos de 10 años. Con las personas que los hermanos entrevistados conviven de manera general se encuentran mamá, papá, tíos, abuelos y hermanos. Es importante reconocer que los hermanos de la muestra (niños diagnosticados con TEA) pertenecen totalmente al sexo masculino.

Tabla 1. Caracterización de la muestra. Hermanos de niños diagnosticados con T.E.A.

\begin{tabular}{|l|c|c|c|c|c|l|} 
Participantes & \multicolumn{7}{c|}{$\begin{array}{c}\text { Sexo del } \\
\text { hermano } \\
\text { diagnosticado } \\
\text { con TEA }\end{array}$} & $\begin{array}{c}\text { Personas con las que } \\
\text { convive en casa }\end{array}$ \\
\hline Hermano 1 & $x$ & & 13 años & $x$ & & Mamá, hermanos y abuelos \\
\hline Hermano 2 & & $x$ & 11 años & $x$ & & Ambos padres, hermano y tía \\
\hline Hermano 3 & & $x$ & 13 años & $x$ & & Padre y hermanos \\
\hline Hermano 4 & & $x$ & 10 años & $x$ & & Ambos padres y hermanos \\
\hline Hermano 5 & $x$ & & 10 años & $x$ & & Ambos padres y hermanos \\
\hline
\end{tabular}

Los padres, madres o tutores que colaboraron a esta investigación cuatro de ellos pertenecen al sexo femenino y uno al sexo masculino, estos se encuentran dentro del rango de edad entre 33- 43 años, encontramos diferentes ocupaciones entre los padres y madres ellas son: estilistas, comerciantes, antropóloga y licenciada en Ciencias Ambientales. El estado civil de los mismos corresponde a tres entrevistados casados, uno soltero y uno separado de la madre de sus hijos. 
Tabla 2. Caracterización de la muestra. Padres, madres o tutores de niños diagnosticados con TEA

\begin{tabular}{|l|l|l|l|}
\hline Participantes & \multicolumn{2}{c|}{ Edad } & \multicolumn{2}{c|}{ Estado civil } \\
\hline Madre 1 & 37 & Estilista & Soltera \\
\hline Madre 2 & 33 & Comerciante & Casada \\
\hline Padre 3 & 40 & Comerciante & Separado \\
\hline Madre 4 & 43 & Antropóloga & Casada \\
\hline Madre 5 & 40 & $\begin{array}{l}\text { Licenciada en Ciencias } \\
\text { Ambientales }\end{array}$ & Casada \\
\hline
\end{tabular}

\section{Percepción acerca de tener un hermano autista}

En el estudio se encontró que los hermanos de niños autistas tienen una percepción de éstos como una persona que asiste a terapia para mejorar sus conductas a la hora de socializar, mejorar la manera de expresar sus emociones, también refieren que con las terapias ellos han aprendido a hablar lo que ha significado un gran logro no solo para el niño sino para la familia. (Tabla 3)

El impacto del diagnóstico del niño con TEA en los hermanos varía de acuerdo a la posición de los hermanos en la familia, existen casos en donde el/la hermano/a mayor experimentó un duelo porque no veía claro el futuro de su hermano, no sabían sobre el trastorno ni cómo manejarlo. En cambio, los hermanos menores o con una edad contemporánea a la del niño autista lo han aprendido a sobre llevar de una manera más natural, experimentando diferentes emociones como celos porque se le brinda mayor atención al niño autista, y también están alerta en las crisis para no salir lastimados. 


\section{Tabla 3. Análisis de la entrevista a hermanos de niños diagnosticados con TEA.}

\begin{tabular}{|c|c|c|}
\hline Pregunta & Categorías & Resultado de la entrevista \\
\hline $\begin{array}{l}\text { ¿Porque su } \\
\text { hermano asiste } \\
\text { a terapia? }\end{array}$ & $\begin{array}{l}\text { Conocimiento } \\
\text { de las terapias } \\
\text { de los hermanos } \\
\text { con TEA. }\end{array}$ & $\begin{array}{l}\text { H1: A mi hermano lo llevaban a terapia } \\
\text { porque él cuando estaba chiquito como de } \\
\text { dos años le gustaba estar en la cuna y a mí } \\
\text { me preguntaban y a mi mama siempre que, } \\
\text { porque le gustaba estar en la cuna, porque } \\
\text { a la mayoría de niños no les gusta y él solo } \\
\text { pasaba meciéndose. } \\
\text { H2: Mi hermano asiste a terapia porque él } \\
\text { tiene un problema, tiene autismo por eso. } \\
\text { H3: Mi hermano asiste a terapia para que } \\
\text { él pueda hablar mejor y los doctores pues le } \\
\text { ayudan a que socialice con más personas. } \\
\text { H4: Mi hermano lo llevan al ClE porque tiene } \\
\text { autismo. } \\
\text { H5: Mi hermano asiste a terapia porque, él } \\
\text { tiene una condición llamada Autismo y ahí } \\
\text { le intentan disminuir su hiperactividad para } \\
\text { luego empezar a enseñarle cosas. }\end{array}$ \\
\hline $\begin{array}{l}\text { ¿Cómo se porta } \\
\text { su hermano con } \\
\text { usted? }\end{array}$ & $\begin{array}{l}\text { Comportamiento } \\
\text { del niño con TEA } \\
\text { y su hermano. }\end{array}$ & $\begin{array}{l}\text { H1: Mi hermano es muy cariñoso conmigo } \\
\text { como ve que yo le doy mucho afecto entonces } \\
\text { anda detrás de mí. } \\
\text { H2: Conmigo mi hermano se porta bien. } \\
\text { H3: Yo pues a veces lo regaño por que el } \\
\text { entiende pues cuando hace algo malo. } \\
\text { H4: Conmigo bueno con todos es normal } \\
\text { pues se porta como pues si bien no es odioso, } \\
\text { ni grosero. } \\
\text { H5: Pues a veces cuando está enojado se } \\
\text { pega en la cabeza a veces le pega con quien } \\
\text { está enojado o si está enojado con alguien, } \\
\text { pero no se quiso desquitar pues va con otro } \\
\text { entonces se pone enojado si no ponemos una } \\
\text { película que a él le gusta que eso es lo que } \\
\text { casi pasa viendo todo el día pues. }\end{array}$ \\
\hline $\begin{array}{l}\text { ¿Cómo se } \\
\text { siente con las } \\
\text { reacciones de } \\
\text { su hermano con } \\
\text { TEA? }\end{array}$ & $\begin{array}{l}\text { Sentimientos } \\
\text { que generan las } \\
\text { reacciones del } \\
\text { niño con TEA al } \\
\text { hermano. }\end{array}$ & $\begin{array}{l}\text { H1: A veces me pongo algo triste por lo que } \\
\text { al niño a veces no lo podemos controlar. } \\
\text { H2: Al principio me sentía preocupada por sus } \\
\text { acciones porque creía que él no iba a hablar, } \\
\text { pero ya al tiempo ya me estoy sintiendo bien } \\
\text { porque él va aprendiendo cada día más y yo } \\
\text { sé que él va a ser una persona normal como } \\
\text { nosotros. }\end{array}$ \\
\hline
\end{tabular}




\begin{tabular}{|l|l|l|}
\hline Pregunta & \multicolumn{1}{|c|}{ Resultado de la entrevista } \\
\hline & $\begin{array}{l}\text { H4: Para mí la primera vez pues fue raro } \\
\text { porque yo decía no es como un niño normal, } \\
\text { pero ya después al pasar el tiempo me fui } \\
\text { acostumbrando. } \\
\text { H5: La mayoría lamentablemente se pega } \\
\text { si está enojado se pega también si esta } \\
\text { frustrado y ese es un problema que tenemos } \\
\text { nosotros que a veces esta tan enojado que } \\
\text { hasta se mueve y pega la cabeza en la pared } \\
\text { más cercana. }\end{array}$ \\
\hline
\end{tabular}

En el estudio se demuestra que los hermanos tienen una percepción positiva a cerca del niño con TEA, también de manera general son conscientes del trastorno de su hermano, y sus diferentes reacciones con las que han aprendido a convivir, pero esto no ha sido motivo para dejar la preocupación por las crisis que ellos atraviesan.

Los datos anteriormente descritos son coherentes con el estudio realizado por Peral Feliz (2013), en donde se llegó a la conclusión de que la experiencia de tener un hermano con autismo difiere en ciertos aspectos de la experiencia de crecer con un hermano con desarrollo típico o con otro tipo de discapacidad. Esta conclusión está basada en el hecho de que los hermanos suelen expresar que lo más difícil para ellos son sobre todo los problemas de comportamiento y las dificultades en la comunicación e interacción social del hermano con autismo.

\section{Rol que ejercen los hermanos en las familias con niños Autistas}

A continuación, este apartado brinda información exclusiva de la entrevista realizada a los padres y madres sobre el rol que ejercen los hermanos en las familias.

El rol que ejerce cada hermano en la vida de un niño autista de las diferentes familias que aplicaron al estudio, se ha encontrado mucha similitud en su desempeño.

Debido a que a los niños autistas una de sus principales dificultades es la interacción social, los hermanos son facilitadores de estas mismas; ejemplificada en la siguiente frase la mamá cuatro relata: "cuando la niña no va por ejemplo al parque y solo va el niño, él decide mejor no ir, y así es para los diferentes lugares en donde salimos. Porque ella es la que sirve como puente para la relación con los demás niños". Sin duda la relación entre los hermanos es una oportunidad para que estos niños desarrollen una comprensión sobre las relaciones sociales. 
Los padres de familia reconocen la importancia de la existencia de un hermano para los niños autistas, algunas de las frases fueron: la mamá cinco manifiesta, "si no tuviera hermano, no sé cómo sería porque él al ver a los hermanos a aprendido cosas imitando, él es muy independiente", mamá uno dijo: "yo creo que si mi hijo con autismo fuera hijo único no tuviera el mismo avance porque a veces entre los mismos hermanos por ley o a fuerza hacen que tengan una socialización mientras cuando es hijo único no".

\section{Tabla 4. Análisis del rol que ejercen los hermanos de niños autistas (madres y padres)}

\begin{tabular}{|c|c|c|}
\hline Pregunta & Categorías & Resultados de la entrevista \\
\hline $\begin{array}{l}\text { ¿Cuál es } \\
\text { el rol que } \\
\text { desempeña } \\
\text { este hermano } \\
\text { en la vida del } \\
\text { niño con TEA? }\end{array}$ & $\begin{array}{l}\text { Rol que } \\
\text { desempeña el } \\
\text { hermano en la } \\
\text { vida de niño con } \\
\text { TEA. }\end{array}$ & $\begin{array}{l}\text { M1: El rol que desempeña mi hijo } \\
\text { mayor en la vida de su hermano es } \\
\text { prácticamente, él dice como papá, } \\
\text { pero en realidad es un hermano mayor } \\
\text { como cuidador, y la niña pequeña } \\
\text { cuando van al parque lo hala para } \\
\text { jugar, pero eso si él no socializa con } \\
\text { otros niños. } \\
\text { M2: Le digo que ella es una mamá } \\
\text { completa es una niña madura y yo } \\
\text { pienso a veces que ella ha madurado a } \\
\text { lo mejor a raíz de eso, porque nos han } \\
\text { pasado varias cosas no crea. } \\
\text { P3: El rol que cumple mi hija es de } \\
\text { mamá, porque ella lo cuida, lo baña, } \\
\text { lo acaricia y es muy afectuosa con él. } \\
\text { M4: Su principal rol con él uno } \\
\text { enseñarle otras cosas que él, o sea } \\
\text { cosas de la vida cotidiana y la miro } \\
\text { yo a ella como una facilitadora en las } \\
\text { relaciones sociales del niño. } \\
\text { M5: El rol que desempeña su hermano } \\
\text { es de cuidador porque él es el que se } \\
\text { encarga de estar pendiente de lo que } \\
\text { el niño necesita o cuando quiere algún } \\
\text { tipo de muñequitos de películas o si } \\
\text { quiere comida o algo entonces es de } \\
\text { cuidador. }\end{array}$ \\
\hline $\begin{array}{l}\text { ¿̇En qué le } \\
\text { ayuda este } \\
\text { hermano al } \\
\text { niño con TEA? }\end{array}$ & $\begin{array}{l}\text { Ayuda del } \\
\text { hermano al niño } \\
\text { con TEA. }\end{array}$ & $\begin{array}{l}\text { M1: La más pequeña le ayuda a } \\
\text { socializar bastante, el otro niño en el } \\
\text { aspecto que como yo trabajo entonces } \\
\text { él a veces me ayuda a cuidar al niño a } \\
\text { mantenerlo más o menos estable. }\end{array}$ \\
\hline
\end{tabular}




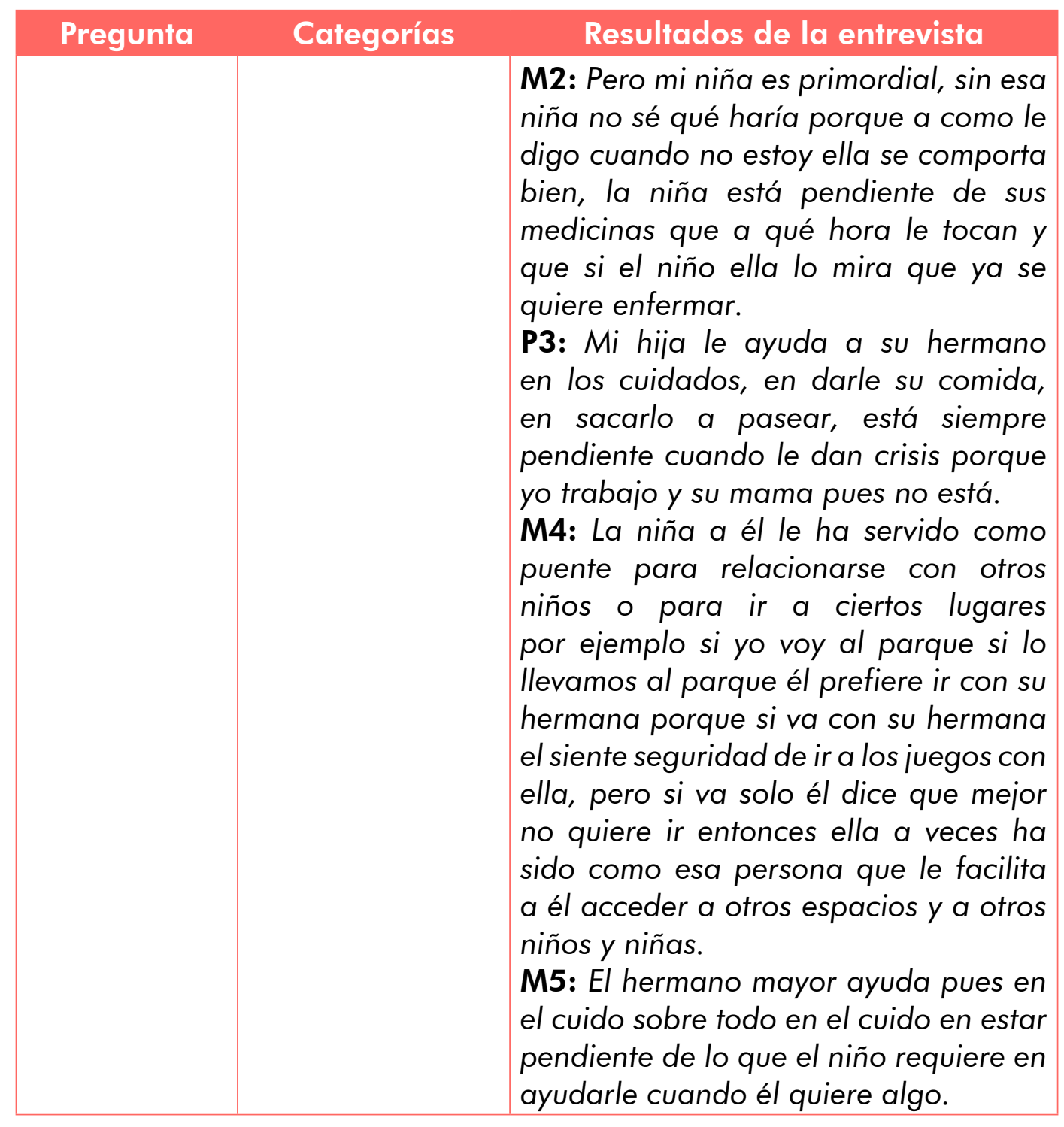

El papel de los hermanos es considerado como primordial para los padres de familia, quienes expresan que los hermanos ejercen el rol de cuidador, ellas mismas dicen que estos viven pendientes de la comida, del bienestar, de sus medicinas, de mantenerlos de alguna manera ocupados por la hiperactividad propias del trastorno, también referían que los hermanos están en vigilancia para evitar cualquier tipo de accidentes ya que ellos no miden el peligro en ningún espacio de su desarrollo.

Los roles de liderazgo, enseñanza, cuidados y ayuda que los hermanos han desarrollado en el proceso de adaptación familiar después del diagnóstico del niño autista, ha generado una idea en los padres de familia de que los hermanos ejercen un papel de segunda mamá o segundo papá en la vida de su niño con TEA.

En el estudio se encontró información relevante acerca de otros factores que inciden en el rol que desempeña el hermano del niño con TEA, entre ellos encontramos: edad, sexo y ausencia de la figura materna o paterna. Dos 
de las hermanas entrevistadas son mayores que el niño con autismo y ellas mismas carecen de una figura materna, también otro hermano que igual es mayor ejerce el papel de protector y cuidador por la ausencia del padre, es por esto que ellos tienden a llevar una mayor responsabilidad sobre sus hermanos y ejercer el papel de mamá y papá.

Por otro lado, los hermanos que viven con mamá y papá la situación son distinta, no ejercen el rol de figura paterna o materna, pero si son cuidadores, protectores y facilitadores en las relaciones sociales de sus hermanos.

Como se explicó anteriormente el hermano del niño autista ejerce un papel importante para su desarrollo en los diferentes ámbitos. Según MeynckensFourez (1999) la relación fraterna ejerce, al menos, tres funciones: apego, suplencia parental y aprendizaje de los papeles sociales y cognitivos. López (1984) señala que el apego persiste a lo largo de la vida y que en la edad adulta se manifiesta por medio del mantenimiento de una relación periódica con los hermanos. Para Milevsky (2005) el apoyo fraterno es compensatorio, sobre todo ante la falta de los padres y amigos. (Meynckens et al., citado por Peral Feliz, 2013)

Para los hermanos de niños autista el cuidarlos y protegerlos ha sido un rol que ellos se han tomado de una manera muy madura a su corta edad, los hermanos entrevistados han expresado que ellos en la vida de su hermano son como protectores en el área social porque ellos han experimentado situaciones en donde ven a su hermano como víctima de discriminación y etiqueta donde ellos se han sentido molestos, tristes y en algún momento frustrado porque las personas no comprenden la condición de su hermano. (Tabla 5)

\section{Tabla 5. Analisis del rol que ejercen los hermanos de niños autistas} (hermanos)

\begin{tabular}{|c|c|c|}
\hline Preguntas & Categorías & Resultados de la entrevista \\
\hline $\begin{array}{l}\text { ¿Qué rol } \\
\text { desempeña } \\
\text { en la vida de } \\
\text { su hermano } \\
\text { con TEA? }\end{array}$ & $\begin{array}{l}\text { Rol que } \\
\text { desempeña } \\
\text { el hermano } \\
\text { en la vida } \\
\text { del niño con } \\
\text { TEA. }\end{array}$ & $\begin{array}{l}\text { H1: Yo siento que en la vida de mi hermano soy casi } \\
\text { el padre porque a mí siempre me toca cuidarlo como } \\
\text { a veces no está mucho tiempo con el papá entonces } \\
\text { yo asumo como esa responsabilidad. } \\
\text { H2: Yo soy su cuidadora porque cuando mi mamá } \\
\text { no está yo estoy pendiente de mi hermano. } \\
\text { H3: En mi actitud siento que soy como la segunda } \\
\text { madre porque ahorita mi mamá no está en el país. } \\
\text { H4: Yo siento que yo para el soy como su protectora, } \\
\text { porque cada vez por ejemplo hay veces que a él } \\
\text { lo molestan solo por eso y pues lo que hago yo es } \\
\text { defenderlo y así, y a mí me molesta eso. } \\
\text { H5: Supongo que el que desempeño yo es ayudar } \\
\text { a mi papa y a mi mama a cuidarlo y cuando este } \\
\text { grande yo protegerlo de las personas que le quieran } \\
\text { hacer mal. }\end{array}$ \\
\hline
\end{tabular}


Otros hermanos cumplen el papel de cuidador, ellos manifiestan que están pendientes de sus medicinas, de lo que les gusta comer o ver en la televisión, que no vallan a la calle solos porque les puede pasar un accidente; en su mayoría los hermanos refieren que ellos son como una segunda mamá o un segundo papá por la ausencia de uno o ambos de los progenitores por motivos de trabajo o porque simplemente no conviven con ellos, por esto han tomado esta responsabilidad.

\section{Repercusiones psicosociales que experimenta el hermano del niño Autista}

Las frases más relevantes en este punto fueron por la hermana dos: "yo tengo que esperar que mi hermano se duerma para poder salir a jugar con mis amigos de la cuadra", "Cuando él llora por algo y no le entiendo que cosa es me hace sentir mal que quiero buscar cómo hacer para ayudarlo", también el hermano cinco relató: "una vez mi hermano llegó con mi papá a recogerme a la escuela y mis compañeros lo quedaron viendo raro por como el actuó entonces yo les explique la condición de mi hermano".

La presencia de niños diagnosticados con TEA ha significado para sus hermanos un cambio tanto familiar como social. Estos hermanos muestran una mayor empatía, tolerancia al estrés, capacidad para adaptarse y mayor sentido de responsabilidad de cuidado de la familia. Las repercusiones psicosociales que experimentan los hermanos varían de acuerdo a cada núcleo familiar.

Las repercusiones se presentan a través de los cambios de actitudes y pensamientos en los hermanos de niños autistas, en las frases anteriores podemos notar la necesidad que los hermanos sienten para proteger de una u otra manera al niño autista en los diferentes medios, lo difícil que es convivir con una persona que no expresa sus emociones de manera verbal y también el cuido que ellos demandan anteponiéndolo a sus gustos y necesidades de recreación.

\section{Test de la familia}

Lo más relevante que prevalece en el test de la familia es: todos los examinados reflejan violencia y audacia como rasgo de su personalidad, dos de ellos presentaron tendencia a replegarse a sí mismo entendiéndose replegar como la idea de emplearse respecto a la persona que opta por no exteriorizar sus emociones, sentimientos o pensamientos, tres representan a través de su dibujo apegarse a las reglas, más de la mitad de la muestra representada por tres examinados presentan personalidad soñador idealista lo que se interpreta que es servicial y leal como amigo para quien las obligaciones son absolutamente sagradas, los sentimientos de los demás son importantes y le encanta hacer feliz a otra gente se siente satisfecho con un pequeño círculo de amigos, sus necesidades de contacto social no son muy marcadas. 
En general, todos los examinados se han adaptado a la vida familiar, cuatro de los hermanos examinados muestran sentimientos de inferioridad e inseguridad, más de la mitad de los hermanos de la muestra demostraron signo de ansiedad evidenciado por el sombreado del dibujo, igual que en el indicador anterior tres de los hermanos muestran signos de ansiedad y conflictos emocionales, desvalorización hacia su persona, hacia sus hermanos y algunos desvalorizan la figura materna al omitirlos en el dibujo de la familia y por último la mayoría de los hermanos presentan perturbación de las relaciones interpersonales. (Ver figura 1)

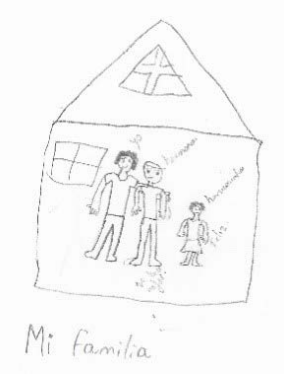

\section{Figura 1. Dibujo de la familia H1}

Estos son los principales datos analizado por la aplicación del test de la familia a la muestra seleccionada los hermanos de niños autistas dándole salida al objetivo específico de la identificación de las repercusiones psicosociales que experimenta el hermano del niño autista. (Ver figura 2 y 3 )

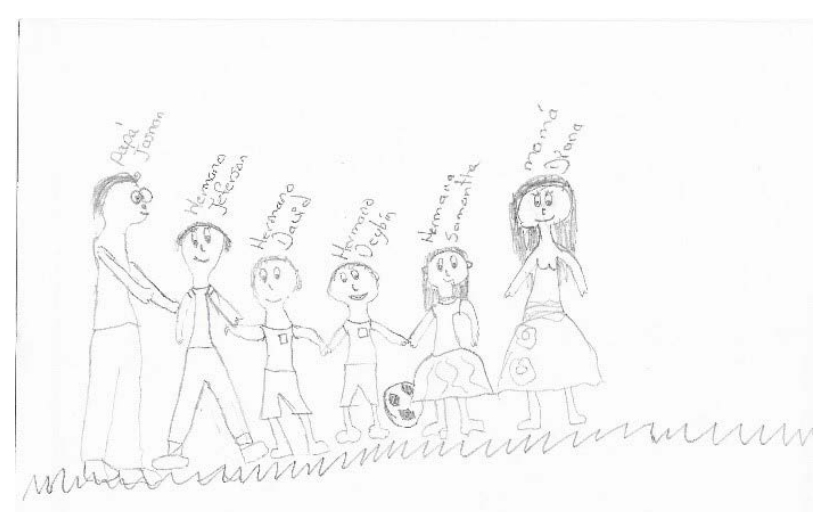

Figura 2. Dibujo de la familia H3 


\section{스교}

Figura 3. Dibujo de la familia H2

Percepciones, roles y repercusiones psicosociales del hermano del niño autista

En la triangulación de los instrumentos aplicados a la muestra (Tabla 6) se presentan elementos relevantes para la investigación, en la percepción que tienen los hermanos de niños con Trastorno del Espectro Autista, como elementos coincidentes entre la entrevista aplicada a hermanos, madres, padres y el test de la familia tenemos: preocupación por el comportamiento y las crisis del niño autista, sobreprotección porque los hermanos siempre quieren saber lo que hace y donde está el niño y preferencia por querer estar siempre junto a su hermanos para cuidarlo y protegerlo.

En el rol que ejercen los hermanos en las familias con niños autistas se encontró como elementos coincidentes: cuidador, protector, facilitador en las relaciones sociales, figura materna, figura paterna, defensor y guía, ellos expresan que siempre están pendiente de las necesidades de su hermano en la ausencia de sus padres, ellos relatan que no lo hacen porque sean mandados por sus padres sino porque ellos sienten la necesidad de cuidarlos.

Finalmente, en las repercusiones psicosociales que experimenta el hermano del niño autista se encontró que para los entrevistados el hecho de tener un hermano autista que demande cuido y protección ha hecho que los prioricen ante sus gustos y necesidades de recreación. 
Tabla 6. Triangulación de instrumentos

\begin{tabular}{|c|c|c|c|c|}
\hline Información & \multicolumn{3}{|c|}{ Instrumentos } & Intersección \\
\hline Categorías & $\begin{array}{c}\text { Entrevista a los } \\
\text { hermanos }\end{array}$ & $\begin{array}{c}\text { Entrevista a } \\
\text { las madres } \\
\text { y padres de } \\
\text { familia }\end{array}$ & Test de la familia & $\begin{array}{l}\text { Elementos } \\
\text { coincidentes }\end{array}$ \\
\hline $\begin{array}{l}\text { Percepción } \\
\text { que tienen } \\
\text { los hermanos } \\
\text { de niños con } \\
\text { Trastorno } \\
\text { del Espectro } \\
\text { Autista. }\end{array}$ & $\begin{array}{l}\text { Los hermanos } \\
\text { de niños } \\
\text { autistas sienten } \\
\text { preocupación } \\
\text { por el } \\
\text { comportamiento } \\
\text { y las crisis de los } \\
\text { mismos. }\end{array}$ & $\begin{array}{l}\text { Los padres } \\
\text { de familias } \\
\text { reconocen que } \\
\text { la relación entre } \\
\text { sus hijos por la } \\
\text { convivencia diría } \\
\text { y el aporte que el } \\
\text { hermano brinda } \\
\text { en el desarrollo } \\
\text { social, transmite } \\
\text { confianza y } \\
\text { seguridad en el } \\
\text { niño autista. }\end{array}$ & $\begin{array}{l}\text { Los examinados } \\
\text { expresaron que } \\
\text { a pesar de las } \\
\text { dificultades que } \\
\text { atraviesa su hermano } \\
\text { día a día a ellos } \\
\text { les gusta compartir } \\
\text { tiempo más que con } \\
\text { otro miembro de la } \\
\text { familia. }\end{array}$ & $\begin{array}{l}\text { Los elementos } \\
\text { coincidentes son los } \\
\text { siguientes: } \\
\text { - Preocupación } \\
\text { - Sobreprotección } \\
\text { - Preferencia }\end{array}$ \\
\hline $\begin{array}{l}\text { Rol que } \\
\text { ejercen los } \\
\text { hermanos en } \\
\text { las familias } \\
\text { con niños } \\
\text { autistas }\end{array}$ & $\begin{array}{l}\text { Los hermanos } \\
\text { expresan que } \\
\text { el rol que ellos } \\
\text { toman en la vida } \\
\text { de su hermano } \\
\text { es de cuidador, } \\
\text { protector y en } \\
\text { otros casos dicen } \\
\text { que ellos son } \\
\text { como la segunda } \\
\text { mamá y el } \\
\text { segundo papá, } \\
\text { porque son ellos } \\
\text { quienes están } \\
\text { pendiente de las } \\
\text { necesidades de } \\
\text { su hermano en } \\
\text { la ausencia de } \\
\text { sus padres, ellos } \\
\text { relatan que no } \\
\text { lo hacen porque } \\
\text { sean mandados } \\
\text { por sus padres } \\
\text { sino porque } \\
\text { ellos sienten la } \\
\text { necesidad de } \\
\text { cuidarlos. }\end{array}$ & $\begin{array}{l}\text { Los padres logran } \\
\text { identificar que } \\
\text { los hermanos } \\
\text { efectivamente } \\
\text { cumplen un rol } \\
\text { en la vida de } \\
\text { su hijo autista, } \\
\text { viéndolo como } \\
\text { un complemento } \\
\text { en su desarrollo, } \\
\text { ellos expresan } \\
\text { que sus hijos son } \\
\text { como cuidadores, } \\
\text { facilitadores de } \\
\text { las relaciones } \\
\text { sociales de su } \\
\text { niño, y en uno } \\
\text { de los casos el } \\
\text { padre dice que su } \\
\text { hija es como una } \\
\text { madre. }\end{array}$ & $\begin{array}{l}\text { En el dibujo de la } \\
\text { familia se evidencia } \\
\text { la necesidad } \\
\text { de cercanía del } \\
\text { examinado hacia } \\
\text { su hermano, } \\
\text { interpretándolo como } \\
\text { una manera de } \\
\text { protección y cuidado } \\
\text { hacia él. }\end{array}$ & $\begin{array}{l}\text { Los elementos } \\
\text { coincidentes en } \\
\text { los instrumentos } \\
\text { aplicados se } \\
\text { encontraron los } \\
\text { siguientes: } \\
\text { - Cuidador } \\
\text { - Protector } \\
\text { - Facilitador en } \\
\text { las relaciones } \\
\text { - sociales } \\
\text { - Figura materna } \\
\text { - Defigura paterna } \\
\text { - Guía }\end{array}$ \\
\hline
\end{tabular}




\begin{tabular}{|c|c|c|c|c|}
\hline Información & \multicolumn{3}{|c|}{ Instrumentos } & Intersección \\
\hline Categorías & $\begin{array}{c}\text { Entrevista a los } \\
\text { hermanos }\end{array}$ & $\begin{array}{c}\text { Entrevista a } \\
\text { las madres } \\
\text { y padres de } \\
\text { familia }\end{array}$ & Test de la familia & $\begin{array}{l}\text { Elementos } \\
\text { coincidentes }\end{array}$ \\
\hline $\begin{array}{l}\text { Repercusiones } \\
\text { psicosociales } \\
\text { que } \\
\text { experimenta el } \\
\text { hermano del } \\
\text { niño autista. }\end{array}$ & $\begin{array}{l}\text { Para los } \\
\text { entrevistados el } \\
\text { hecho de tener } \\
\text { un hermano } \\
\text { autista que } \\
\text { demande cuido } \\
\text { y protección ha } \\
\text { hecho que los } \\
\text { prioricen ante } \\
\text { sus gustos y } \\
\text { necesidades de } \\
\text { recreación. }\end{array}$ & $\begin{array}{l}\text { Los padres de } \\
\text { familia expresan } \\
\text { que en muchas } \\
\text { ocasiones el niño } \\
\text { con TEA si no } \\
\text { está su hermano } \\
\text { presente en } \\
\text { actividades fuera } \\
\text { del hogar el niño } \\
\text { prefiere no asistir, } \\
\text { puesto que el } \\
\text { hermano le da } \\
\text { seguridad. }\end{array}$ & $\begin{array}{l}\text { El test de la } \\
\text { familia refleja } \\
\text { que los hermanos } \\
\text { presentan signos de } \\
\text { ansiedad y conflictos } \\
\text { emocionales lo que } \\
\text { indica dificultad en } \\
\text { la comunicación } \\
\text { familiar, también } \\
\text { se encontró } \\
\text { desvalorización hacia } \\
\text { algunos miembros } \\
\text { de la familia } \\
\text { interpretándose como } \\
\text { un mecanismo de } \\
\text { defensa hacia la } \\
\text { persona con quien } \\
\text { no tiene afinidad } \\
\text { y que en lo último } \\
\text { el sujeto desea su } \\
\text { desaparición. }\end{array}$ & $\begin{array}{l}\text { Dentro de los } \\
\text { elementos } \\
\text { coincidentes } \\
\text { encontramos los } \\
\text { siguientes: } \\
\text { - Priorizar } \\
\text { - Insatisfecho } \\
\text { - Estrés }\end{array}$ \\
\hline
\end{tabular}

\section{Estrategias de intervención psicológicas que resulten efectivas para la adaptación y disminución del estrés en el entorno familiar de los hermanos de niños autistas}

En los hermanos de niños diagnosticados con Trastorno del Espectro Autista se identificó adaptación en su entorno familiar pero también cierto nivel de estrés por las preocupaciones sobre las crisis que en ocasiones presentan los niños, el cuido que requieren y la responsabilidad que los padres de manera indirecta les otorgan, a través de los distintos instrumentos aplicados como la entrevista en profundidad y el dibujo de la familia.

En la entrevista aplicada a los padres se obtuvo información complementaria para la elaboración de las estrategias de intervención psicológica que se puede utilizar con los hermanos de las cuales algunas son específicas para trabajar con niños con TEA, pero estas se tomaron en cuenta para que el hermano tenga otras herramientas que ayuden en la evolución del niño autista desde casa.

La importancia de esta propuesta tiene como fin mejorar la adaptación y disminuir el estrés causado por las diferentes reacciones propias del Trastorno del Espectro Autista, que serán aplicadas en los diferentes centros de atención de niños diagnosticados con TEA de la ciudad de Estelí. 
A continuación, matriz de estrategia de intervención psicológica aplicada a hermanos de niños diagnosticados con Trastorno del Espectro Autista.

Tabla 7. Propuesta de intervención psicológica para la adaptación y disminución del estrés en el entorno familiar de los hermanos de niños autistas

Objetivo general: Proponer a los diferentes centros en donde atienden niños diagnosticados con TEA nuevas estrategias de intervención psicológica que ayuden a la adaptación y disminución del estrés de los hermanos.

\begin{tabular}{|c|c|c|c|}
\hline Objetivo especifico & Puntos a trabajar & $\begin{array}{l}\text { Actuaciones de los } \\
\text { centros }\end{array}$ & $\begin{array}{l}\text { Técnicas de } \\
\text { intervención }\end{array}$ \\
\hline $\begin{array}{l}\text { Crear un espacio } \\
\text { confortable para } \\
\text { que los hermanos } \\
\text { compartan } \\
\text { sentimientos, } \\
\text { intercambien } \\
\text { experiencia, brinden } \\
\text { apoyo mutuo y } \\
\text { aprender a resolver } \\
\text { y enfrentar las } \\
\text { situaciones que les } \\
\text { genere estrés. }\end{array}$ & $\begin{array}{l}\text { Exteriorizar su vivencia } \\
\text { familiar a través del } \\
\text { relato en el grupo. }\end{array}$ & $\begin{array}{l}\text { Crear el espacio en los } \\
\text { diferentes centros con el } \\
\text { terapeuta del mismo en } \\
\text { días intercalados lunes, } \\
\text { miércoles y viernes. }\end{array}$ & Grupo de apoyo \\
\hline $\begin{array}{l}\text { Establecer el } \\
\text { funcionamiento del } \\
\text { sistema familiar que } \\
\text { favorezca los retos } \\
\text { y demandas de la } \\
\text { convivencia en las } \\
\text { familias con niños } \\
\text { diagnosticados con } \\
\text { TEA. }\end{array}$ & $\begin{array}{l}\text { - Mejorar el vínculo } \\
\text { familiar. } \\
\text { - Que los padres } \\
\text { conozcan el sentir } \\
\text { de los hiijos que no } \\
\text { tienen el trastorno. } \\
\text { - Mejorar la } \\
\text { comunicación entre } \\
\text { los miembros de la } \\
\text { familia. }\end{array}$ & $\begin{array}{l}\text { Los terapeutas de cada } \\
\text { centro deberán aplicar las } \\
\text { diferentes técnicas propias } \\
\text { de la terapia sistémica } \\
\text { familiar. } \\
\text { - Terapia estructural } \\
\text { - Terapia narrativa } \\
\text { - Terapia cognitivo- } \\
\text { conductual }\end{array}$ & Terapia familiar \\
\hline $\begin{array}{l}\text { Mejorar el bienestar } \\
\text { de los hermanos de } \\
\text { niños con TEA }\end{array}$ & $\begin{array}{l}\text { Disminuir los niveles } \\
\text { de ansiedad y } \\
\text { preocupación. }\end{array}$ & $\begin{array}{l}\text { Los terapeutas de cada } \\
\text { centro deberían de aplicar } \\
\text { Terapia de relajación de } \\
\text { Jacobson. }\end{array}$ & $\begin{array}{l}\text { Terapia de } \\
\text { relajación }\end{array}$ \\
\hline
\end{tabular}


Objetivo general: Proponer a los diferentes centros en donde atienden niños diagnosticados con TEA nuevas estrategias de intervención psicológica que ayuden a la adaptación y disminución del estrés de los hermanos.

\begin{tabular}{|c|c|c|c|}
\hline Objetivo especifico & Puntos a trabajar & $\begin{array}{l}\text { Actuaciones de los } \\
\text { centros }\end{array}$ & $\begin{array}{l}\text { Técnicas de } \\
\text { intervención }\end{array}$ \\
\hline $\begin{array}{l}\text { Dar a conocer } \\
\text { herramientas a } \\
\text { los hermanos } \\
\text { para desarrollar } \\
\text { habilidades que } \\
\text { ayuden al niño con } \\
\text { TEA. }\end{array}$ & $\begin{array}{l}\text { Conocer las estrategias } \\
\text { de la terapia de juego }\end{array}$ & $\begin{array}{l}\text { Los terapeutas podrían } \\
\text { enseñarles a los hermanos } \\
\text { las diferentes técnicas de la } \\
\text { terapia de juego de manera } \\
\text { sencilla para que le ayude } \\
\text { a la evolución del niño y } \\
\text { fortalezcan los vínculos } \\
\text { entre ellos. Las técnicas } \\
\text { propuestas son: } \\
\text { - Juego de roles } \\
\text { - Juegos imitación } \\
\text { - Ejercicio de soplo } \\
\text { - Danza y el movimiento } \\
\text { - El dibujo, la pintura o } \\
\text { plastilina. }\end{array}$ & Terapia de juego \\
\hline $\begin{array}{l}\text { Desarrollar destrezas } \\
\text { en el hermano neuro } \\
\text { típico que le ayude } \\
\text { a la estimulación del } \\
\text { niño con TEA. }\end{array}$ & $\begin{array}{l}\text { - Estimulación. } \\
\text { - Fomentar la } \\
\text { - Autonomía. } \\
\text { - } \text { oportación al } \\
\text { - Facilitar la } \\
\text { comprensión del } \\
\text { entorno. } \\
\text { - Aportar confianza y } \\
\text { seguridad. }\end{array}$ & $\begin{array}{l}\text { Desarrollar talleres } \\
\text { vivenciales en donde el } \\
\text { hermano aprenda nuevas } \\
\text { herramientas de acuerdo } \\
\text { a la metodología Teacch } \\
\text { para luego aplicarlas en el } \\
\text { hogar. }\end{array}$ & $\begin{array}{l}\text { Metodología } \\
\text { Teacch }\end{array}$ \\
\hline
\end{tabular}

\section{CONCLUSIONES}

En el trabajo investigativo se encontró que los hermanos de niños autistas tienen una percepción positiva acerca de ellos expresando que lo ven como una persona que asiste a terapia para mejorar sus conductas a la hora de socializar, mejorar la manera de expresar sus emociones también refieren que con las terapias ellos han aprendido a hablar lo que ha significado un gran logro no solo para el niño sino para la familia. Se pudo evidenciar que en general la muestra es consciente del trastorno de su hermano, y sus diferentes reacciones con las que han aprendido a convivir, pero esto no ha sido motivo para dejar la preocupación por las crisis que estos atraviesan. 
Los padres de familia afirman que el rol que ejerce cada hermano en la vida de un niño autista en las diferentes familias se ha encontrado similitud en el desempeño. Para estos padres y madres el papel de los hermanos es considerado como primordial ellos expresan que estos hermanos ejercen el rol de cuidador, también refieren que estos hermanos están en vigilancia para evitar cualquier tipo de accidentes ya que ellos no miden el peligro en ningún espacio de su desarrollo, estas acciones del hermano han generado una idea en los padres de familia de que estos ejercen un papel de segunda mamá o segundo papá en la vida de su niño con TEA.

Por otro lado, para los hermanos de niños autista el cuidarlos y protegerlos ha sido un rol que ellos se han tomado de una manera muy madura, los hermanos entrevistados han expresado que ellos en la vida de su hermano son como protectores. Otros cumplen el papel de cuidador estos manifiestan que están pendientes de sus medicinas, de lo que les gusta comer o ver en la televisión.

Para los hermanos de niños autistas no todo ha sido negativo a pesar del cambio que ha significado el nuevo diagnostico en la familia, la adaptación al nuevo estilo de vida y la aceptación de la condición de su hermano. Estos muestran una mayor empatía, tolerancia al estrés, capacidad para adaptarse y mayor sentido de responsabilidad de cuidado de la familia. Las repercusiones psicosociales que experimentan los hermanos varían de acuerdo a cada núcleo familiar.

Los principales efectos psicológicos y sociales de los hermanos de niños autistas van en dependencia del impacto del diagnóstico. En general todos los examinados se han adaptado a la vida familiar, sin embargo, muestran sentimiento de inferioridad e inseguridad relacionado a la falta de atención por parte de los padres ya que el hermano autista demanda más cuidados y por último la mayoría de los hermanos presentan perturbación de las relaciones interpersonales.

Se concluye que la vivencia de los hermanos de niños con TEA en su mayoría cumple el rol de cuidador, protector y facilitador de las relaciones sociales. También se identificó que los padres y madres de familia han dado mucha responsabilidad a sus hijos sobre el cuido a su hermano con autismo, expresando ellos mismo que su hijo/a cumple el papel de mamá o papá. La mayoría de los hermanos que ejercen el rol de mamá o papá en el niño autista es por la ausencia de la figura paterna o materna, este dato es uno de los factores que más inciden en el apego y sobre protección hacia el hermano autista.

En el proceso de investigación se percibieron ciertas limitaciones para continuar con el estudio, por ejemplo, en un mismo centro no se encontró la cantidad de familias previstas que cumplieran con los criterios de la muestra, es por esta razón que se decidió realizar a nivel municipal y no de un solo centro. 


\section{REFERENCIAS BIBLIOGRÁFICAS}

Freixa Niella, M. (1993). Familia y deficiencia mental. Realidades, necesidades y recursos de los hermanos. Universidad de Barcelona, Barcelona, España. Obtenido de https://www.tdx.cat/bitstream/handle/10803/2361/03. MFN_3de8.pdf? sequence $=3$ \&isAllowed $=y$

Gonzalez Gonzalez, R. (2018). proyecteo de apoyo a hermanos de niños diagnosticados con Trastorno de espectro autista. Fomentar el vinculo fraterno y la relacion en el seno de la familia. Valencia, España.

Hernandez Sampieri, R., Fernandez Collado, C., \& Baptista Lucio, P. (2007). metodología de la investigacion. México, México: Ultra.

Kids Health. (marzo de 2019). Obtenido de https://kidshealth.org/es/ parents/pervasive-develop-disorders-esp.html

Martínez Martín, M. Á., \& Bilbao León, M. C. (2008). Acercamiento a la realidad de las familias de personas con autismo. (C. O. Madrid, Ed.) Psychosocial Intervention, 17(2), 215-230. Obtenido de https://www. redalyc.org/pdf/1798/179814018009.pdf

Meneu, M. A. (15 de octubre de 2019). Psicologia online. Obtenido de https://www.psicologia-online.com/el-test-de-la-familia-2606.html

Peral Feliz, S. (2013). Hermanos de personas con Trastorno del Espectro Autista. Universidad de Salamanca. Salamanca, España: Universidad de Salamanca. Obtenido de file:///C:/Users/pc/Downloads/30-8-55-33. admin.Hermanos_de_personas_con_TEA.pdf

Perez Castañeda, C. G., \& Verdugo Alonso, M. A. (2006). La influencia de un hermano con autismo sobre la calidad de vida familiar. Revista Española sobre Discapacidad Intelectual, 39(227), 75- 90. Obtenido de http:// riberdis.cedd.net/handle/11181/3805

Piura Lopez, J. (2000). Introduccion a la Metodologia de la Investigacion Cientifica. Managua, Nicaragua: Nuevo Amanecer. 